\title{
Interpolation of Histological Slices by Means of Non-Rigid Registration
}

\author{
Simone Gaffling ${ }^{1,2}$, Florian Jäger ${ }^{1,2}$, Volker Daum ${ }^{1}$, Miyuki Tauchi ${ }^{3}$, \\ Elke Lütjen-Drecoll ${ }^{3}$ \\ ${ }^{1}$ Lehrstuhl für Mustererkennung \\ ${ }^{2}$ SAOT Graduate School in Advanced Optical Technologies \\ ${ }^{3}$ Institut für Anatomie, Lehrstuhl II \\ Friedrich-Alexander-Universität Erlangen-Nürnberg \\ simone.gaffling@informatik.uni-erlangen.de
}

\begin{abstract}
It is a common approach to create and inspect histological slices to investigate functional and morphological structures on a cellular level. For the easier analysis of the resulting data sets, the underlying 3 -D structure has to be reconstructed and visualized. Due to mechanical stress imposed on the tissue during the slicing, some slices are damaged, leading to an unsatisfying reconstruction result. In this article we present a means to interpolate missing images. For this, a deformation field, calculated by a variational non-rigid registration method using adjacent slices as reference and template images, is partially applied to the template image. The approach is tested on a histological data set, and evaluated using visual inspection by experts. The resulting reconstruction is shown to be a significant improvement.
\end{abstract}

\section{Introduction}

3 -D visualization of images is advantageously applied in a broad range of areas, especially for medical imaging modalities such as CT, MRI and Ultrasound. In functional morphology, however, the majority of images that is dealt with are still 2-D histological images. A problem is that in some cases it would be benefitial to be able to relate the displayed cellular features to the underlying 3-D structure of the tissue. As a consequence, several attempts are recently made to perform 3-D visualization of histological slices. For instance, the Neuroscanning Project of the Institute for Anatomy, University of Lübeck, aims to reconstruct human and animal brains based on series of high-resolution histological images. Their goal is to be able to remodel the neural distribution in the brain [1]. Furthermore, Weinhold et al. [2] reconstruct a series of histological slices of a rat liver, to be able to understand the capability of the liver to regenerate.

However, the data sets that have to be dealt with are afflicted with artifacts, stemming from the mechanical stress that was imposed on the tissue during the cutting of the slices and the following staining process, which includes exposure to heat, liquids and chemicals. As a result, the final digital images show artifacts 
like holes, blurred regions, and different inter-slice intensities. In particular, some slices are completely disrupted and cannot be used for reconstruction (Fig.1).

To our knowledge, no approach to deal with the problem of defect slices was proposed before. For a good 3-D reconstruction, though, complete data sets are a must, and depending on the specific case, a total loss of $5-30 \%$ of the images is possible. This is the reason why missing slices have to be interpolated. This work adresses this issue, by presenting a method to interpolate those images, and the performance of the proposed method is demonstrated on a histological data set.

\section{Methods}

The interpolation of images is performed by means of non-rigid registration. A deformation field between two neighboring slices is calculated, and the missing step(s) are created by partial application of the deformation field on one of the images. The non-rigid registration technique of choice is a variational approach, as proposed in [3].

\subsection{Preprocessing}

The digital images acquired from the histological slices show many artifacts that have to be taken into account, including intensity differences between slices, which makes an intensity normalization obligatory. Here, histogram matching provided by the Insight Toolkit (ITK) [4] is incorporated. After a gray-level conversion, a reference slice for the normalization is determined, and the associated image histogram is then used as reference to linearly match the histograms of the other slices, resulting in same intensities for same features. Afterwards, a rigid registration of all images is performed using an ITK multilevel rigid registration approach [4] to align the displayed tissue for the whole data set.

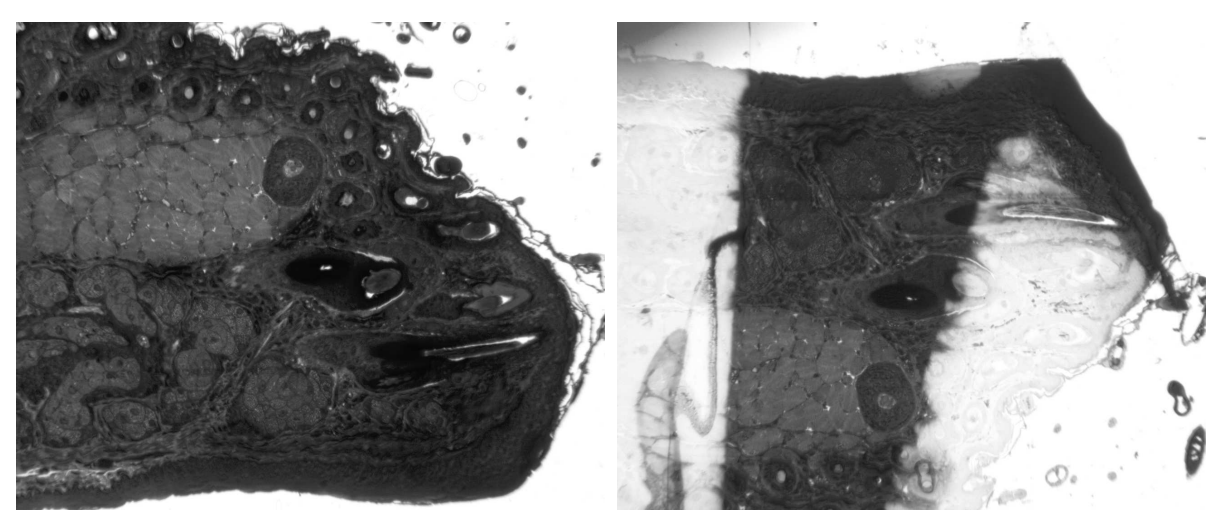

Fig. 1. Left: Intact histological slice. Right: Disrupted slice unsuitable for reconstruction. 


\subsection{Variational approach}

After the preprocessing steps, the images adjacent to a missing slice are used as reference $R$ and template image $T$, and a deformation field $u$ transforming $T$ to $R$ is calculated, i.e. $R=T_{u}=T(x-u(x))$. The parameters of the previously performed rigid registration are used as initialization, and the following registration is performed on an overlapping region of both images, to prevent unrealistic deformations of the edges. The non-rigid registration method of choice is the variational formulation of the problem, introduced in [3]. The objective function to be minimized is given by

$$
\mathcal{J}[u]:=\mathcal{D}[R, T ; u]+\alpha \mathcal{S}[u] \rightarrow \min ,
$$

where $\mathcal{D}[R, T ; u]$ is a distance metric used to evaluate the similarity between the reference image and the transformed template image. We choose mutual information (MI), measuring the amount of information one image contains about the other image. The second term $\mathcal{S}[u]$, called regularizer, is weighted by an adjustable parameter $\alpha$, and keeps the deformation field smooth.

In general, an optimization problem of this kind is solved by calculating the Gâteaux-derivatives of the individual terms, and finding a root of the resulting equation. Hermosillo et al. [5] developed an expression for the gradient of the mutual information, with respect to a deformation field. For the estimation of the joint intensity probability that is used for the computation of the MI, Parzen windowing is applied, using a Gaussian kernel with $\sigma=2.0$. As regularizer, the curvature regularizer as proposed in [3] is used. Also, in contrast to [3], an inexact Newton method is employed in the nonlinear optimization.

\subsection{Interpolation}

After performing the beforementioned steps, the missing slices are eventually interpolated. This avoids holes in the final reconstruction, cmp. Fig. 3. The deformation field $u$ resulting from the non-rigid registration is divided into several parts, depending on the number of missing slices $k$ in the respective gap. The final deformation field that has to be applied to the template image $T$ to approximate the i-th intermediate slice $S_{i}$ is then given by

$$
u_{i}=\frac{i}{k+1} \cdot u,
$$

and the interpolated slice itself is given by application on the template image, $S_{i}=T_{u_{i}}$.

\subsection{Evaluation strategies}

The approach was tested using a histological data set consisting of 42 images, with a size of $1300 \times 1030$ pixels. The images represent a tissue sample cut into 60 slices, where 18 were lost in the cutting process and unsuitable for further 
processing. An expert working prevalently with histological images was asked to inspect the results. The first task was to find out whether interpolated images can be identified if they are randomly mixed with original histological images. At the same time the quality of the images should be rated on a scale from 1 (very good) to 5 (very bad), deciding whether the images show the features of interest in a realistic way. Last, the smoothness of transition from reference to template image should be rated, where the image series consisted of the reference image, the template image, and the interpolated slices in between.

\section{Results}

Showing the results to an expert, $36.67 \%$ of the unaltered histological images and $50 \%$ of the interpolated images were correctly identified as original resp. interpolated images, and their overall quality was denoted to be good (2.1 resp. 2.0). For the smoothness of transition in an image series of five images ( $\mathrm{R}$, three interpolated, $\mathrm{T}$ ), the interpolated series was graded with 2.0 , where the original series was found to be very good (1.0). For two smaller image series consisting of three images, the interpolated series were found to be better (2.0 resp. 1.0) than the original series (3.0 resp. 2.0).

Fig. 2 compares our approach to a linear interpolation between two slices. On the left detail image showing the linear interpolation, the displayed structures are visible twice, leading to a blurred appearance. In contrast to that, the slice on the right hand side, interpolated by non-rigid registration, shows the structures only once, and in a more natural way.

Fig. 3 shows the reconstruction of a hair follicle contained in the histological data set. On the left hand, the original data set with missing images is shown. The parts where images are missing are clearly visible. On the right hand side, the same structure, reconstructed using interpolated images, is shown.
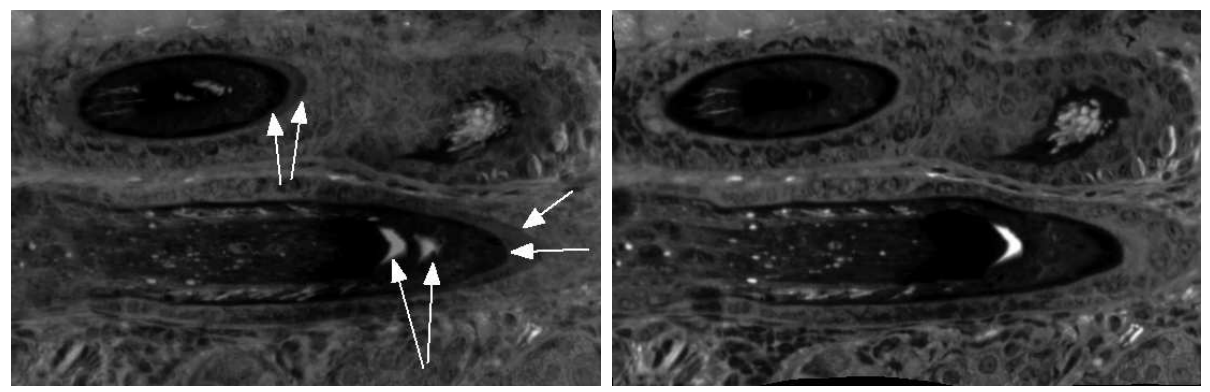

Fig. 2. Details of an interpolation. Left: Linear interpolation between $R$ and $T$. Right: Our approach. 
Fig. 3. Reconstructed hair follicle: original (left), interpolated (right

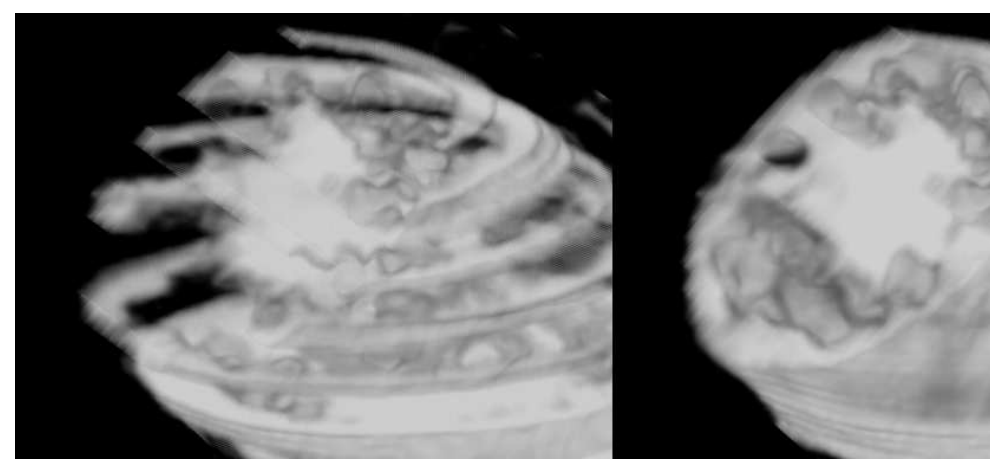

\section{Discussion}

It turned out that the interpolation of missing slices works well if the parameters of the non-rigid registration are chosen carefully. The overall quality of the interpolated images was found satisfying by experts, similar to the quality of the original images, and the reconstruction is significantly improved if missing slices were interpolated.

One drawback is the relatively large "gap" between the reference and first interpolated slice in the case of images with large differences, which requires further refinement of the approach. Still, the result prooves to be a more thorough and superior 3-D reconstruction than without the interpolated images.

For the future, an important task is to further evaluate the approach on other data sets. In addition, non-rigid registration of all slices of the data sets should be incorporated, to correct deformations of the tissue introduced during the cutting of the histological slices.

\section{References}

1. Schmitt O, Modersitzki J, Heldmann S, et al. Image registration of sectioned brains. Int J Computer Vis. 2007;73:5-39.

2. Weinhold A, Wirtz S, Schenk A, et al. Vollautomatische Vorverarbeitung und rigide Registrierung zur Rekonstruktion von Bildern histologischer Stufenschnitte der Rattenleber. Procs BVM. 2007; p. 419-423.

3. Modersitzki J. Numerical Methods for Image Registration. Oxford University Press; 2003.

4. Ibanez L, Schroeder W, Ng L, et al. The ITK Software Guide. 2nd ed. Kitware, Inc; 2005.

5. Hermosillo G. Variational Methods for Multimodal Image Matching. University of Nice Sophia Antipolis; 2002. 